\title{
DETERMINATION OF X-RAY TOTAL ATTENUATION COEFFICIENT IN Zr, Ag, In FOR ENERGY RANGE BETWEEN 10.5-111.9 keV
}

\author{
Ü. Turgut, E. BÜYÜKKasap, Ö. ŞimşEK, M. ERTuĞRUl and O. DoĞaN \\ Atatürk University, Education Faculty, Department of Physics Education \\ 25240 Erzurum, Turkey
}

(Received December 4, 1997; revised version March 2, 1998)

The total X-ray attenuation coefficients in elements $\mathrm{Zr}, \mathrm{Ag}$, and In for $K_{\alpha}$ and $K_{\beta}$ characteristic lines of elements with different $Z(33 \leq Z \leq 92)$ were estimated by measuring attenuation in transmission method. The intensity of $K$ X-rays was monitored by a $\mathrm{Ge}(\mathrm{Li})$ detector with energy resolution $190 \mathrm{eV}$ at $5.9 \mathrm{keV}$. The experimentally measured attenuation coefficients were found in good agreement with the theoretical values of attenuation coefficients calculated by Storm, Israel and Hubbell, Seltzer.

PACS numbers: $32.80 .-\mathrm{t}$

\section{Introduction}

For many X-ray based techniques the knowledge of X-ray attenuation coefficients is an effectual value limiting the precision of the method. The total attenuation coefficients are important atomic data of interest both in fundamental physics and in many applied fields. The experimental measurements of the total attenuation coefficients have been performed by several investigators [1-3]. A great number of experimental data have been obtained in a wide energy range and tabulated in publications [4]. We measured total attenuation coefficients in $\mathrm{Ti}, \mathrm{Fe}, \mathrm{Ni}$, $\mathrm{Zn}$ for X-ray energy region between 4.5-111.9 keV [5]. Theoretical values of the total attenuation coefficients were calculated and tabulated $[6,7]$. Certain discrepancies in currently available experimental data have caused an upsurge of interest in precise total attenuation coefficients measurements.

The present work describes the determination of the total attenuation coefficients in $\mathrm{Zr}, \mathrm{Ag}$, and In for X-ray energy region between $10.5-111.9 \mathrm{keV}$ by using transmission method.

\section{Experimental}

The X-ray total attenuation coefficients were determined by measuring transmission of the fluorescent $K$ characteristic lines with known energy through targets of known thickness. The experimental arrangement is shown in Fig. 1. Secondary targets of elements with different $Z(33 \leq Z \leq 92)$ were irradiated, in turn, with 


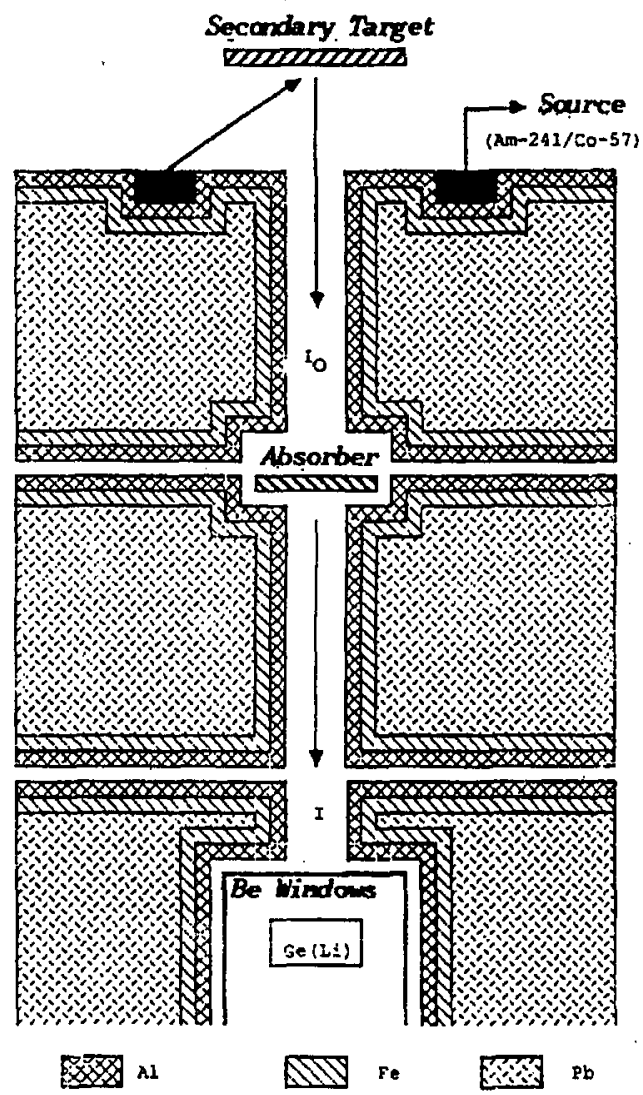

Fig. 1. Experimental arrangement geometry.

$59.57 \mathrm{keV}$ gamma rays from ${ }^{241} \mathrm{Am}$ source (for $Z \leq 65$ elements) and $122 \mathrm{keV}$ gamma rays from $10 \mathrm{mCi}{ }^{57} \mathrm{Co}$ sources (for $Z>65$ elements). The emitted $K$ fluorescent $\mathrm{X}$-rays were collimated by the lead collimator shielded with aluminium and iron to fall on the absorbers of $\mathrm{Zr}, \mathrm{Ag}$, and $\mathrm{In}$ cut from their high purity (99.00-99.99\%) foils. The thicknesses of the absorbers were $25 \mu \mathrm{m}$. This thickness, $t$, satisfies the condition $\mu t<1$, where $\mu$ is an attenuation coefficient. Thus, effects of multi-scattered photons in the targets were reduced. To minimise the effect of small-angle scattering in the absorber, the transmitted $K \mathrm{X}$-rays were further collimated by the lead collimator shielded with aluminium and iron and counted using a $\mathrm{Ge}(\mathrm{Li})$ detector with energy resolution $190 \mathrm{eV}$ at $5.9 \mathrm{keV}$. A typical spectrum is given in Fig. 2.

Spectra were recorded with and without the absorber for different energies of $K$ fluorescent lines. For some target-absorber combinations used in the present measurements (e.g. Zr target, $\mathrm{Zr}$ absorber) the target and absorber spectra overlap. The fluorescent $\mathrm{X}$-rays produced in the absorber by the $59.57 \mathrm{keV}$ gamma rays scattered from the secondary target were also counted together with the sec- 


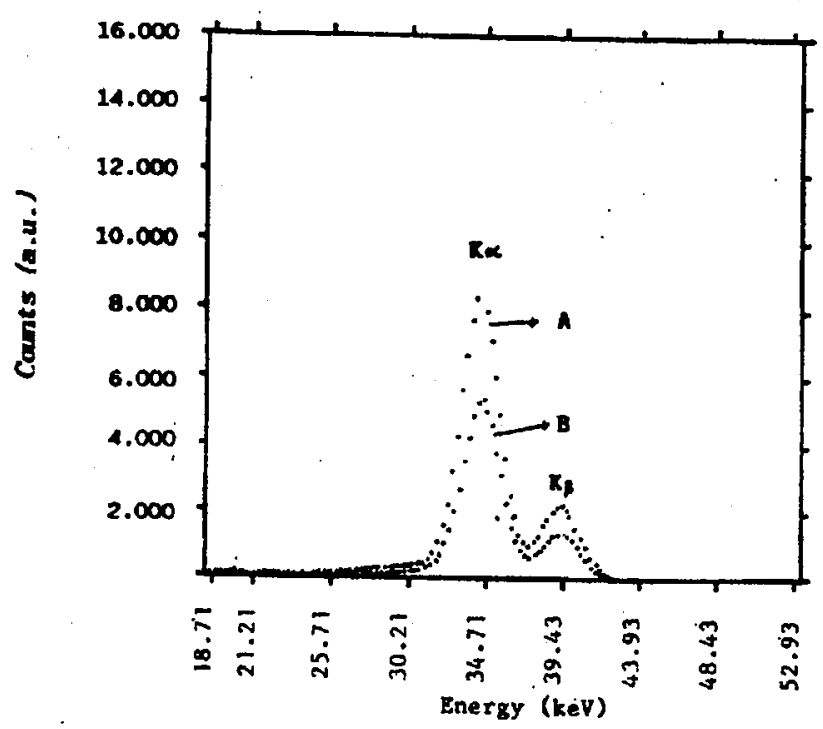

Fig. 2. X-rays spectra of $\mathrm{Ce} K$ characteristic lines. $A$ - without absorber and $B-$ with $\mathrm{Ag}$ absorber.

ondary target fluorescent X-rays transmitted through the absorber. The measured attenuation coefficients therefore need to be corrected for the contribution to the absorber fluorescent intensity of the $\mathrm{X}$-rays produced by gamma rays scattered from the target. This contribution was measured experimentally by replacing the experimental target with equivalent $\mathrm{Al}$ target which does not produce characteristic fluorescent lines in this range of energy, only scatter radiation and spectra were corrected for this contribution. Average energies for $K_{\alpha}$ and $K_{\beta}$ characteristic lines of elements were taken from Storm and Israel [6].

The counts for the measurements of each $\mathrm{X}$-ray group were taken in the following sequence: no absorber $\left(I_{0}\right)$, absorber $(I)$. In an ideal transmission experiment, the photon once scattered in the absorber, even at very small angle, should not be detected. We assumed that in the experimental arrangement we satisfied this condition.

Total linear attenuation coefficients $\left(\mu_{l}\right)\left[\mathrm{cm}^{-1}\right]$ of elements were calculated from the following equation obtained from $I=I_{0} \exp \left(-\mu_{l} t\right)$ :

$$
\mu_{l}=-\frac{1}{t}\left(\ln \frac{I}{I_{0}}\right)
$$

where $t(\mathrm{~cm})$ is the thickness of target; $I$ and $I_{0}$ are areas under the peak for the absorber and no absorber respectively.

Total attenuation coefficients $\sigma_{\mathrm{T}}$ (barns/atom) were calculated by using the total linear attenuation coefficients from the following equation:

$$
\sigma_{\mathrm{T}}=10^{24} \frac{\mu_{l}}{\rho} \frac{M}{N_{0}}
$$


where $\mu_{l}$ are total linear attenuation coefficients in $\mathrm{cm}^{-1} ; \rho\left(\mathrm{g} / \mathrm{cm}^{3}\right)$ is the density of element; $\mu_{l} / \rho$ are the mass attenuation coefficients in $\mathrm{cm}^{2} / \mathrm{g}, M$ is the atomic mass of element and $N_{0}$ is the Avogadro number. $N_{0} / M$ describes numbers of atoms per gram. $10^{24}$ factor comes from 1 barn equal to $10^{-24} \mathrm{~cm}^{2}$.

\section{Results and discussion}

The experimental values of the total attenuation coefficients are listed together with the theoretical values of Storm and Israel [6] and Hubbell and Seltzer [7] in Table. These values were presented in Figs. 3-5. The uncertainties in the values of the total attenuation coefficients are estimated approximately $<5 \%$. These are due to counting statistics $<1 \%$, thickness determination $<1 \%$, scattering contribution $<2 \%$ and peak area determination $<1 \%$. As shown in Table and Figs. $3-5$, our experimental values are in good agreement with the theoretical values.

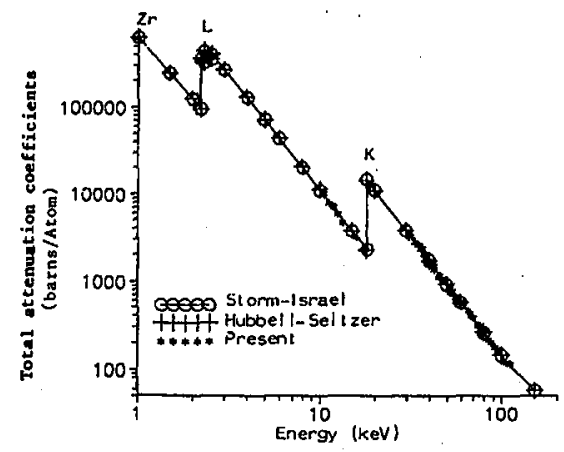

Fig. 3. Comparison of present experimental values and theoretical values for $\mathrm{Zr}$.

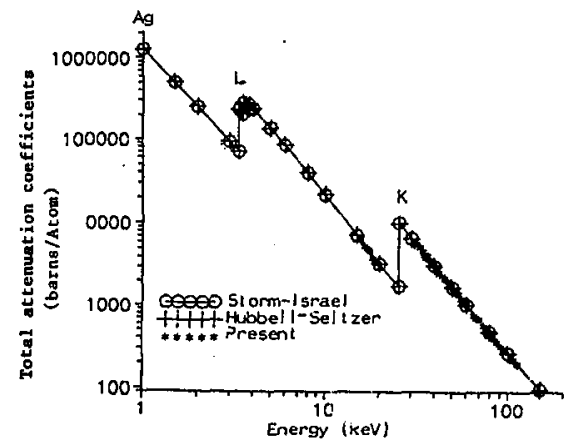

Fig. 4. Comparison of present experimental values and theoretical values for Ag. 


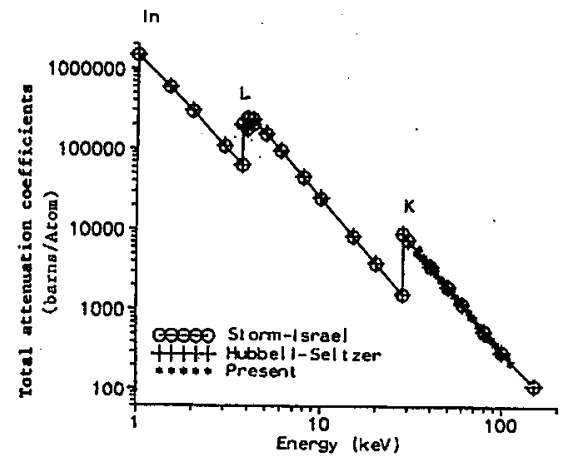

Fig. 5. Comparison of present experimental values and theoretical values for In.

TABLE

Total attenuation coefficients in $\mathrm{Zr}, \mathrm{Ag}$, and In in barns/atom.

\begin{tabular}{|c|c|c|c|c|c|c|c|c|}
\hline \multicolumn{3}{|c|}{ Secondary exciter } & \multicolumn{2}{|c|}{$\mathrm{Zr}$} & \multicolumn{2}{|c|}{$\mathrm{Ag}$} & \multicolumn{2}{|c|}{ In } \\
\hline & Lines & $\begin{array}{c}\text { Energy } \\
{[\mathrm{keV}]}\end{array}$ & Present & Theor. & Present & Theor. & Present & Theor. \\
\hline As & $K_{\alpha}$ & 10.532 & $\begin{array}{l}9981 \\
\pm 210\end{array}$ & $\begin{array}{l}9570[6] \\
9758[7]\end{array}$ & - & - & - & - \\
\hline As & $K_{\beta}$ & 11.730 & $\begin{array}{l}7321 \\
\pm 130\end{array}$ & $\begin{array}{l}7164[6] \\
7280[7]\end{array}$ & - & - & - & - \\
\hline $\mathrm{Se}$ & $K_{\alpha}$ & 11.210 & $\begin{array}{l}7818 \\
\pm 150\end{array}$ & $\begin{array}{l}8093[6] \\
8236[7]\end{array}$ & - & - & - & - \\
\hline $\mathrm{Se}$ & $K_{\beta}$ & 12.503 & $\begin{array}{c}5878 \\
\pm 120\end{array}$ & $\begin{array}{l}6035[6] \\
6120[7]\end{array}$ & - & - & - & - \\
\hline $\mathrm{Br}$ & $K_{\alpha}$ & 11.907 & $\begin{array}{r}7026 \\
\pm 140\end{array}$ & $\begin{array}{l}6882[6] \\
6990[7]\end{array}$ & - & - & - & - \\
\hline $\mathrm{Zr}$ & $K_{\alpha}$ & 15.746 & $\begin{array}{l}3129 \\
\pm 65\end{array}$ & $\begin{array}{l}3239[6] \\
3270[7]\end{array}$ & $\begin{array}{r}6324 \\
\pm 125\end{array}$ & $\begin{array}{l}6194[6] \\
6280[7]\end{array}$ & - & - \\
\hline $\mathrm{Zr}$ & $K_{\beta}$ & 17.700 & $\begin{array}{l}2284 \\
\pm 45\end{array}$ & $\begin{array}{l}2351[6] \\
2379[7]\end{array}$ & $\begin{array}{l}4576 \\
\pm 95\end{array}$ & $\begin{array}{l}4518[6] \\
4576[7]\end{array}$ & - & - \\
\hline $\mathrm{Nb}$ & $K_{\alpha}$ & 16.584 & - & - & $\begin{array}{l}5326 \\
\pm 110\end{array}$ & $\begin{array}{l}5386[6] \\
5340[7]\end{array}$ & - & - \\
\hline $\mathrm{Nb}$ & $K_{\beta}$ & 18.661 & - & - & $\begin{array}{l}3769 \\
\pm 75\end{array}$ & $\begin{array}{l}3918[6] \\
3967[7]\end{array}$ & $\begin{array}{c}4762 \\
\pm 90\end{array}$ & $\begin{array}{l}4629[6] \\
4699[7]\end{array}$ \\
\hline
\end{tabular}


TABLE (cont.)

Total attenuation coefficients in $\mathrm{Zr}, \mathrm{Ag}$, and In in barns/atom.

\begin{tabular}{|c|c|c|c|c|c|c|c|c|}
\hline \multicolumn{3}{|c|}{ Secondary exciter } & \multicolumn{2}{|c|}{$\mathrm{Zr}$} & \multicolumn{2}{|c|}{$\mathrm{Ag}$} & \multicolumn{2}{|c|}{ In } \\
\hline & Lines & $\begin{array}{c}\text { Energy } \\
{[\mathrm{keV}]}\end{array}$ & Present & Theor. & Present & Theor. & Present & Theor. \\
\hline Mo & $K_{\alpha}$ & 17.443 & - & - & $\begin{array}{l}4817 \\
\pm 100\end{array}$ & $\begin{array}{l}4700[6] \\
4659[7]\end{array}$ & $\begin{array}{l}5153 \\
\pm 110\end{array}$ & $\begin{array}{l}5553[6] \\
5639[7]\end{array}$ \\
\hline Mo & $K_{\beta}$ & 19.648 & - & - & - & - & $\begin{array}{l}4028 \\
\pm 80\end{array}$ & $\begin{array}{l}4028[6] \\
4088[7]\end{array}$ \\
\hline $\mathrm{Te}$ & $K_{\beta}$ & 31.103 & $\begin{array}{l}3492 \\
\pm 75\end{array}$ & $\begin{array}{l}3401[6] \\
3413[7]\end{array}$ & $\begin{array}{l}5534 \\
\pm 120\end{array}$ & $\begin{array}{l}5964[6] \\
5974[7]\end{array}$ & - & - \\
\hline Cs & $K_{\alpha}$ & 30.851 & $\begin{array}{l}3187 \\
\pm 60\end{array}$ & $\begin{array}{l}3476[6] \\
3490[7]\end{array}$ & $\begin{array}{l}5947 \\
\pm 125\end{array}$ & $\begin{array}{l}6093[6] \\
6104[7]\end{array}$ & - & - \\
\hline Cs & $K_{\beta}$ & 35.131 & $\begin{array}{l}2534 \\
\pm 40\end{array}$ & $\begin{array}{l}2445[6] \\
2453[7]\end{array}$ & $\begin{array}{l}4138 \\
\pm 100\end{array}$ & $\begin{array}{l}4324[6] \\
4335[7]\end{array}$ & $\begin{array}{l}4748 \\
\pm 75\end{array}$ & $\begin{array}{l}5004[6] \\
5000[7]\end{array}$ \\
\hline $\mathrm{Ba}$ & $K_{\alpha}$ & 32.062 & $\begin{array}{l}3245 \\
\pm 55\end{array}$ & $\begin{array}{l}3132[6] \\
3143[7]\end{array}$ & $\begin{array}{l}5669 \\
\pm 105\end{array}$ & $\begin{array}{l}5504[6] \\
5515[7]\end{array}$ & - & - \\
\hline $\mathrm{Ba}$ & $K_{\beta}$ & 36.535 & $\begin{array}{l}2335 \\
\pm 40\end{array}$ & $\begin{array}{l}2196[6] \\
2206[7]\end{array}$ & $\begin{array}{l}4118 \\
\pm 75\end{array}$ & $\begin{array}{l}3899[6] \\
3911[7]\end{array}$ & $\begin{array}{l}4647 \\
\pm 95\end{array}$ & $\begin{array}{l}4515[6] \\
4517[7]\end{array}$ \\
\hline $\mathrm{La}$ & $K_{\alpha}$ & 33.297 & $\begin{array}{l}2756 \\
\pm 60\end{array}$ & $\begin{array}{l}2827[6] \\
2837[7]\end{array}$ & $\begin{array}{l}5111 \\
\pm 90\end{array}$ & $\begin{array}{l}4982[6] \\
4993[7]\end{array}$ & $\begin{array}{l}5223 \\
\pm 110\end{array}$ & $\begin{array}{l}5759[6] \\
5745[7]\end{array}$ \\
\hline $\mathrm{La}$ & $K_{\beta}$ & 37.966 & $\begin{array}{l}2047 \\
\pm 45\end{array}$ & $\begin{array}{l}1981[6] \\
1988[7]\end{array}$ & $\begin{array}{l}3619 \\
\pm 70\end{array}$ & $\begin{array}{l}3523[6] \\
3534[7]\end{array}$ & $\begin{array}{l}4184 \\
\pm 90\end{array}$ & $\begin{array}{l}4082[6] \\
4088[7]\end{array}$ \\
\hline $\mathrm{Ce}$ & $K_{\alpha}$ & 34.564 & $\begin{array}{l}2576 \\
\pm 50\end{array}$ & $\begin{array}{l}2555[6] \\
2563[7]\end{array}$ & $\begin{array}{l}4604 \\
\pm 80\end{array}$ & $\begin{array}{l}4514[6] \\
4525[7]\end{array}$ & $\begin{array}{l}5905 \\
\pm 120\end{array}$ & $\begin{array}{l}5222[6] \\
5215[7]\end{array}$ \\
\hline $\mathrm{Ce}$ & $K_{\beta}$ & 39.431 & $\begin{array}{l}1697 \\
\pm 30\end{array}$ & $\begin{array}{l}1788[6] \\
1794[7]\end{array}$ & $\begin{array}{l}3249 \\
\pm 55\end{array}$ & $\begin{array}{l}3188[6] \\
3199[7]\end{array}$ & $\begin{array}{l}3526 \\
\pm 80\end{array}$ & $\begin{array}{l}3696[6] \\
3706[7]\end{array}$ \\
\hline $\operatorname{Pr}$ & $K_{\alpha}$ & 35.858 & $\begin{array}{l}2282 \\
\pm 45\end{array}$ & $\begin{array}{l}2313[6] \\
2321[7]\end{array}$ & $\begin{array}{l}3859 \\
\pm 85\end{array}$ & $\begin{array}{l}4097[6] \\
4108[7]\end{array}$ & $\begin{array}{l}4628 \\
\pm 95\end{array}$ & $\begin{array}{l}4742[6] \\
4741[7]\end{array}$ \\
\hline $\operatorname{Pr}$ & $K_{\beta}$ & 40.930 & $\begin{array}{l}1648 \\
\pm 30\end{array}$ & $\begin{array}{l}1614[6] \\
1620[7]\end{array}$ & $\begin{array}{l}2770 \\
\pm 50\end{array}$ & $\begin{array}{l}2885[6] \\
2896[7]\end{array}$ & $\begin{array}{l}3308 \\
\pm 70\end{array}$ & $\begin{array}{l}3348[6] \\
3358[7]\end{array}$ \\
\hline $\mathrm{Nd}$ & $K_{\alpha}$ & 37.179 & $\begin{array}{l}2046 \\
\pm 40\end{array}$ & $\begin{array}{l}2097[6] \\
2104[7]\end{array}$ & $\begin{array}{l}3645 \\
\pm 70\end{array}$ & $\begin{array}{l}3724[6] \\
3711[7]\end{array}$ & $\begin{array}{l}4153 \\
\pm 80\end{array}$ & $\begin{array}{l}4313[6] \\
4317[7]\end{array}$ \\
\hline $\mathrm{Nd}$ & $K_{\beta}$ & 42.460 & $\begin{array}{l}1361 \\
\pm 25\end{array}$ & $\begin{array}{l}1458[6] \\
1465[7]\end{array}$ & $\begin{array}{l}2553 \\
\pm 45\end{array}$ & $\begin{array}{l}2613[6] \\
2624[7]\end{array}$ & $\begin{array}{l}2861 \\
\pm 60\end{array}$ & $\begin{array}{l}3035[6] \\
3043[7]\end{array}$ \\
\hline $\mathrm{Sm}$ & $K_{\alpha}$ & 39.906 & $\begin{array}{l}1693 \\
\pm 35\end{array}$ & $\begin{array}{l}1731[6] \\
1736[7]\end{array}$ & $\begin{array}{l}3081 \\
\pm 65\end{array}$ & $\begin{array}{l}3089[6] \\
3100[7]\end{array}$ & $\begin{array}{l}3729 \\
\pm 65\end{array}$ & $\begin{array}{l}3582[6] \\
3593[7]\end{array}$ \\
\hline $\mathrm{Sm}$ & $K_{\beta}$ & 45.622 & $\begin{array}{l}1119 \\
\pm 25\end{array}$ & $\begin{array}{l}1196[6] \\
1202[7]\end{array}$ & $\begin{array}{l}2199 \\
\pm 44\end{array}$ & $\begin{array}{l}2152[6] \\
2164[7]\end{array}$ & $\begin{array}{l}2537 \\
\pm 45\end{array}$ & $\begin{array}{l}2504[6] \\
2510[7]\end{array}$ \\
\hline
\end{tabular}


TABLE (cont.)

Total attenuation coefficients in $\mathrm{Zr}, \mathrm{Ag}$, and In in barns/atom.

\begin{tabular}{|c|c|c|c|c|c|c|c|c|}
\hline \multicolumn{3}{|c|}{ Secondary exciter } & \multicolumn{2}{|c|}{$\mathrm{Zr}$} & \multicolumn{2}{|c|}{$\mathrm{Ag}$} & \multicolumn{2}{|c|}{ In } \\
\hline & Lines & $\begin{array}{c}\text { Energy } \\
{[\mathrm{keV}]}\end{array}$ & Present & Theor. & Present & Theor. & Present & Theor. \\
\hline \multirow[t]{2}{*}{$\mathrm{Gd}$} & $K_{\alpha}$ & 42.390 & 1494 & $1431[6]$ & 2411 & $2565[6]$ & 3069 & $2980[6]$ \\
\hline & & & \pm 30 & $1437[7]$ & \pm 50 & $2577[7]$ & \pm 65 & $2988[7]$ \\
\hline \multirow[t]{2}{*}{$\mathrm{Gd}$} & $K_{\beta}$ & 48.918 & 1027 & $986[6]$ & 1716 & $1782[6]$ & 1958 & $2078[6]$ \\
\hline & & & \pm 15 & $993[7]$ & \pm 35 & 1794 [7] & \pm 40 & 2082 [7] \\
\hline \multirow[t]{2}{*}{ Dy } & $K_{\alpha}$ & 45.714 & 1176 & $1189[6]$ & 2076 & $2140[6]$ & 2411 & $2491[6]$ \\
\hline & & & \pm 20 & $1196[7]$ & \pm 43 & $2152[7]$ & \pm 50 & 2497 [7] \\
\hline \multirow[t]{2}{*}{ Dy } & $K_{\beta}$ & 52.352 & 746 & $819[6]$ & 1436 & $1485[6]$ & 1718 & $1732[6]$ \\
\hline & & & \pm 15 & $824[7]$ & \pm 30 & $1494[7]$ & \pm 36 & $1735[7]$ \\
\hline \multirow[t]{2}{*}{ Ho } & $K_{\alpha}$ & 47.242 & 1072 & $1086[6]$ & 2019 & $1958[6]$ & 2331 & $2281[6]$ \\
\hline & & & \pm 20 & $1093[7]$ & \pm 45 & $1970[7]$ & \pm 50 & $2286[7]$ \\
\hline \multirow[t]{2}{*}{ Ho } & $K_{\beta}$ & 54.123 & 776 & $748[6]$ & 1411 & $1358[6]$ & 1539 & $1584[6]$ \\
\hline & & & \pm 14 & $752[7]$ & \pm 25 & $1365[7]$ & \pm 30 & $1587[7]$ \\
\hline \multirow[t]{2}{*}{$\operatorname{Er}$} & $K_{\alpha}$ & 48.801 & 1017 & $992[6]$ & 1828 & $1794[6]$ & 1958 & 2091 [6] \\
\hline & & & \pm 23 & 999 [7] & \pm 35 & $1806[7]$ & \pm 43 & 2096 [7] \\
\hline \multirow[t]{2}{*}{$\operatorname{Er}$} & $K_{\beta}$ & 55.930 & 662 & $684[6]$ & 1219 & $1244[6]$ & 1331 & $1450[6]$ \\
\hline & & & \pm 15 & $688[7]$ & \pm 25 & $1249[7]$ & \pm 35 & $1452[7]$ \\
\hline \multirow[t]{2}{*}{$\mathrm{Yb}$} & $K_{\alpha}$ & 52.014 & 828 & $833[6]$ & 1541 & $1511[6]$ & 1828 & $1762[6]$ \\
\hline & & & \pm 20 & 839 [7] & \pm 32 & $1520[7]$ & \pm 40 & 1766 [7] \\
\hline \multirow[t]{2}{*}{$\mathrm{Yb}$} & $K_{\beta}$ & 59.652 & 580 & $574[6]$ & 1065 & $1046[6]$ & 1175 & $1219[6]$ \\
\hline & & & \pm 11 & $576[7]$ & \pm 18 & $1049[7]$ & \pm 20 & $1221[7]$ \\
\hline \multirow[t]{2}{*}{$\mathrm{Ta}$} & $K_{\alpha}$ & 57.078 & 633 & 647 [6] & 1131 & $1178[6]$ & 1411 & $1373[6]$ \\
\hline & & & \pm 14 & $650[7]$ & \pm 20 & $1182[7]$ & \pm 30 & $1375[7]$ \\
\hline \multirow[t]{2}{*}{$\mathrm{Ta}$} & $K_{\beta}$ & 65.529 & 451 & $444[6]$ & 763 & $811[6]$ & 967 & $947[6]$ \\
\hline & & & \pm 10 & $447[7]$ & \pm 13 & $814[7]$ & \pm 20 & $948[7]$ \\
\hline \multirow[t]{2}{*}{ W } & $K_{\alpha}$ & 58.832 & 561 & $596[6]$ & 1050 & $1086[6]$ & 1296 & $1265[6]$ \\
\hline & & & \pm 10 & $599[7]$ & \pm 21 & $1089[7]$ & \pm 30 & $1268[7]$ \\
\hline \multirow[t]{2}{*}{ W } & $K_{\beta}$ & 67.564 & 423 & $409[6]$ & 708 & $747[6]$ & 858 & $872[6]$ \\
\hline & & & \pm 8 & $411[7]$ & \pm 18 & $749[7]$ & \pm 15 & $873[7]$ \\
\hline \multirow[t]{2}{*}{$\mathrm{Au}$} & $K_{\alpha}$ & 68.133 & 395 & $400[6]$ & 697 & $730[6]$ & 841 & $853[6]$ \\
\hline & & & \pm 7 & $402[7]$ & \pm 13 & $733[7]$ & \pm 20 & $854[7]$ \\
\hline \multirow[t]{2}{*}{$\mathrm{Au}$} & $K_{\beta}$ & 78.367 & 256 & $273[6]$ & 474 & $500[6]$ & 607 & $586[6]$ \\
\hline & & & \pm 4 & $276[7]$ & \pm 10 & $498[7]$ & \pm 15 & $586[7]$ \\
\hline
\end{tabular}


TABLE (cont.)

Total attenuation coefficients in $\mathrm{Zr}, \mathrm{Ag}$, and In in barns/atom.

\begin{tabular}{|c|c|c|c|c|c|c|c|c|}
\hline \multicolumn{3}{|c|}{ Secondary exciter } & \multicolumn{2}{|c|}{$\mathrm{Zr}$} & \multicolumn{2}{|c|}{$\mathrm{Ag}$} & \multicolumn{2}{|c|}{ In } \\
\hline & Lines & $\begin{array}{c}\text { Energy } \\
{[\mathrm{keV}]}\end{array}$ & Present & Theor. & Present & Theor. & Present & Theor. \\
\hline \multirow[t]{2}{*}{$\mathrm{Hg}$} & $K_{\alpha}$ & 70.103 & 380 & $370[6]$ & 690 & $676[6]$ & 812 & $780[6]$ \\
\hline & & & \pm 8 & $372[7]$ & \pm 15 & $678[7]$ & \pm 17 & $791[7]$ \\
\hline \multirow[t]{2}{*}{$\mathrm{Hg}$} & $K_{\beta}$ & 80.656 & 265 & $253[6]$ & 472 & $463[6]$ & 481 & $542[6]$ \\
\hline & & & \pm 5 & $255[7]$ & \pm 9 & $465[7]$ & \pm 12 & $542[7]$ \\
\hline \multirow[t]{2}{*}{$\mathrm{Pb}$} & $K_{\alpha}$ & 74.159 & 310 & $317[6]$ & 563 & $581[6]$ & 660 & $679[6]$ \\
\hline & & & \pm 6 & $320[7]$ & \pm 13 & $583[7]$ & \pm 15 & $678[7]$ \\
\hline \multirow[t]{2}{*}{$\mathrm{Pb}$} & $K_{\beta}$ & 85.370 & 211 & $218[6]$ & 387 & $398[6]$ & 454 & $465[6]$ \\
\hline & & & \pm 4 & $220[7]$ & \pm 8 & $400[7]$ & \pm 10 & $467[7]$ \\
\hline \multirow[t]{2}{*}{$\mathrm{Bi}$} & $K_{\alpha}$ & 76.246 & 305 & $294[6]$ & 517 & $539[6]$ & 610 & $630[6]$ \\
\hline & & & \pm 7 & $297[7]$ & \pm 12 & $541[7]$ & \pm 13 & $630[7]$ \\
\hline \multirow[t]{2}{*}{$\mathrm{Bi}$} & $K_{\beta}$ & 87.796 & 198 & $202[6]$ & 353 & $369[6]$ & 449 & $431[6]$ \\
\hline & & & \pm 4 & 204 [7] & \pm 8 & $371[7]$ & \pm 10 & $433[7]$ \\
\hline \multirow[t]{2}{*}{ Th } & $K_{\alpha}$ & 92.050 & 177 & $179[6]$ & 314 & $325[6]$ & 390 & $379[6]$ \\
\hline & & & \pm 2 & $181[7]$ & \pm 6 & $328[7]$ & \pm 8 & $382[7]$ \\
\hline \multirow[t]{2}{*}{$\mathrm{Th}$} & $K_{\beta}$ & 106.169 & 119 & $127[6]$ & 217 & $225[6]$ & 277 & $261[6]$ \\
\hline & & & \pm 2 & $127[7]$ & \pm 4 & $227[7]$ & \pm 6 & $264[7]$ \\
\hline \multirow[t]{2}{*}{$\mathbf{U}$} & $K_{\alpha}$ & 96.977 & 159 & $156[6]$ & 278 & $283[6]$ & 322 & $329[6]$ \\
\hline & & & \pm 3 & $158[7]$ & \pm 5 & $286[7]$ & \pm 6 & $333[7]$ \\
\hline \multirow[t]{2}{*}{$\mathrm{U}$} & $K_{\beta}$ & 111.898 & 116 & $111[6]$ & 195 & $198[6]$ & 223 & $229[6]$ \\
\hline & & & \pm 3 & $113[7]$ & \pm 4 & $200[7]$ & \pm 5 & $232[7]$ \\
\hline
\end{tabular}

\section{References}

[1] N. Singh, H.S. Aulakh, K.L. Allawadhi, B.S.S. Sood, Pramana 33, 505 (1989).

[2] S.C. Lingam, T.S. Babu, V.P. Kumar, D.V.K. Reddy, Can. J. Phys. 67, 139 (1989).

[3] L. Gerward, Nucl. Instrum. Methods Phys. Res. B 69, 407 (1992).

[4] J.H. Hubbell, N. Singh, B. Chand, P.N. Trehan, D. Mehta, M.L. Garg, R.R. Garg, S. Singh, S. Puri, J. Phys. Chem. Ref. Data 23, 2 (1994).

[5] Ü. Turgut, E. Büyükkasap, Ö. Şimşek, M. Ertuğrul, O. Doğan, J. Radioanal. Nucl. Chem. 218, 267 (1997).

[6] E. Storm, I. Israel, Nucl. Data A 7, 565 (1970).

[7] J.H. Hubbell, S.M. Seltzer, Laboratory Report, U.S. Department of Commerce Technology Administration, National Institute of Standards and Technology Physics Laboratory, NISTIR-5632(1995). 